\title{
The peripheral neutrophils in subjects with COPD-0SA overlap syndrome and severe comorbidities: A feasible inflammatory biomarker?
}

\author{
Madalina Macrea ${ }^{1,2, A-F}$, Sabrina Campbell ${ }^{1,3, B}$, Thomas Martin ${ }^{1,2, E, F}$, Kris Ann Oursler ${ }^{1,3, A, C, E, F}$ \\ 1 Salem Veterans Affairs Medical Center, USA \\ 2 Department of Medicine, University of Virginia School of Medicine, Charlottesville, USA \\ ${ }^{3}$ Virginia Tech Carilion School of Medicine, Roanoke, USA \\ A - research concept and design; B - collection and/or assembly of data; C - data analysis and interpretation; \\ $D$ - writing the article; $E$ - critical revision of the article; $F$ - final approval of the article
}

\section{Address for correspondence}

Madalina Macrea

E-mail:madalina.macrea@va.gov

Funding sources

This research was supported by the Department of Veteran Affairs, Veterans Health Administration, Rehabilitation Research and Development Service through the VA Career Development Award given to Dr. Macrea.

Conflict of interest

None declared

Received on August 25, 2016

Reviewed on December 25, 2016

Accepted on July 14, 2017

\begin{abstract}
Background. Overlap syndrome (OS) describes the association of obstructive sleep apnea (OSA) and chronic obstructive pulmonary disease (COPD) in a single individual. Subjects with OS have increased cardiovascular mortality which is presumed to be inflammation-mediated. As a clinical biomarker, an increased neutrophil count correlates with the severity of coronary artery stenosis.
\end{abstract}

Objectives. As little is known about the role of neutrophils in the underlying inflammatory mechanisms in OS, we aimed to assess the percentage of peripheral neutrophils (PPN) in OS vs in COPD alone.

Material and methods. A cross-sectional study of patients with COPD and severe comorbidities, as defined by a Care Assessment Need score over 95, were seen in the Pulmonary Tele-Health Clinic at the Salem Veteran Affairs Medical Center, USA, over a 1-year period. Demographic and polysomnographic data, FEV1 and the Charlson Comorbidity Index (CCI) were extracted from the Electronic Medical Records. Obstructive sleep apnea was defined according to the American Academy of Sleep Medicine (AASM) guidelines. Serum inflammatory markers (PPN, CRP, fibrinogen and procalcitonin) were obtained after the Tele-Health appointment.

Results. Out of the 38 subjects with COPD, 17 (44\%) had OS. Compliance with continuous positive airway pressure therapy (CPAP) was excellent in 705 subjects (41\%). There was a significant difference in the PPN of subjects with OS vs COPD alone, regardless of whether they were compliant $(p=0.03)$ with the CPAP therapy or not $(p=0.005)$. No differences in the severity of COPD, baseline comorbidity, smoking, or inflammatory markers were found between the OS and COPD-only subjects. Body mass index (BMI), COPD severity, smoking, and home oxygen therapy (HOT) use were not associated with PPN ( $p>0.2)$.

Conclusions. Overlap syndrome subjects have higher PPN than those with COPD alone, regardless of their CPAP compliance. Our results could be used to motivate 0 S subjects to improve their lifestyles and to comply with drug therapies aimed at reducing cardiovascular disease (CVD).

Key words: obstructive sleep apnea, chronic obstructive pulmonary disease, cardiovascular, systemic inflammation, neutrophils

DOI

10.17219/acem/75904

\section{Copyright}

Copyright by Author(s)

This is an article distributed under the terms of the

Creative Commons Attribution Non-Commercial License

(http://creativecommons.org/licenses/by-nc-nd/4.0/) 


\section{Introduction}

Chronic obstructive pulmonary disease (COPD), a disease characterized by irreversible airflow limitation, and obstructive sleep apnea disease (OSA), a disease characterized by intermittent collapse of the upper airway, are each responsible for major morbidity, mortality and economic burden. ${ }^{1}$ The term "overlap syndrome" (OS) was introduced to describe the association of both conditions in a single patient. Given the high prevalence of both COPD and OSA, it is expected that up to $29 \%$ of OSA subjects could be affected by OS. ${ }^{2}$ Although the evidence suggests that the increased mortality in OS subjects is primarily cardiovascular, the exact mechanisms remain unclear. ${ }^{1}$ It is likely, though, that cell-mediated inflammation plays an important role given the shared systemic inflammation pathways in COPD, OSA and atherosclerosis. ${ }^{3}$ The possible biological pathways by which neutrophils might influence the development of cardiovascular disease (CVD) are protean and include an increased adherence of platelets to subendothelial collagen and the release of leukotriene, superoxide and inflammatory mediators. ${ }^{4-6}$ As a clinical biomarker, increased neutrophil count correlates with the severity of coronary artery stenosis and survival in subjects with stable coronary artery disease and demonstrates good specificity as a prognostic marker for acute coronary syndrome when the electrocardiogram was nonspecific. ${ }^{7,8} \mathrm{McNicholas} \mathrm{suggested} \mathrm{that} \mathrm{COPD} \mathrm{and}$ OSA are systemic disorders that can cause or worsen CVD, one of the mechanisms related to the chronic intermittent hypoxia that stimulates the inflammatory cytokines in which neutrophils participate. ${ }^{3}$ Despite these findings, the abovedescribed findings were not demonstrated in OS and little is known about the role of neutrophils in the underlying mechanisms of increased mortality in these subjects. We therefore aimed to assess peripheral neutrophils in OS subjects compared to COPD-only subjects with a similar degree of comorbidity. In addition, as it is has been suggested that the current standard therapy for OSA, i.e., continuous positive airway pressure (CPAP), could alter the inflammatory markers in OSA, we evaluated the inflammatory response in both CPAP-compliant and noncompliant OS subjects as compared to COPD-only subjects.

\section{Material and methods}

The study is a cross-sectional retrospective evaluation of subjects with COPD and severe comorbidities selected from subjects followed for routine primary care at the community-based outpatient clinics (CBOC) satellite to the Salem Veterans Affairs Medical Center (VAMC), USA, over 1 year. The severity of comorbidity was assessed by the Care Assessment Need Score (CAN), which indicates how a given patient compares to other individuals in terms of the likelihood of hospitalization or death. Higher CAN scores reflect an increased number of healthcare providers, medications and mental health diagnoses. ${ }^{9}$ Subjects with a CAN score greater than 95 were eligible for the study and their charts were screened for a pulmonary function test (PFT) that showed COPD according to American Thoracic Society (ATS) guidelines. Three hundred and fifty-three subjects with a CAN score over 95 were identified in the CBOC; 150 of these subjects were diagnosed with COPD. Thirty-eight of the COPD subjects were evaluated in the Pulmonary Tele-Health Clinic, out of which 17 had a diagnosis of OSA prior to this study and were defined as cases of OS. Demographic, clinical and medication data (inhaled and oral corticosteroid) were extracted from the Electronic Medical Records (EMR). The Charlson Comorbidity Index (CCI) and the Framingham $(\mathrm{FH})$ 10-year cardiovascular risk score were calculated based on EMR data. ${ }^{10,11}$ Dyspnea severity was assessed using the Medical Research Council Scale. ${ }^{12}$ The study was approved by the Salem VAMC Review Board as quality improvement data.

Among the COPD participants, a chart review was performed for a diagnosis of OSA. The inclusion of OSA subjects was done based on a diagnostic polysomnogram completed prior to our study (SensorMedics, Sunnyvale, USA). The stages of sleep were identified using 2-channel electroencephalogram (C4-A1, C3-A2), chin electromyogram, and left and right electrooculograms. Thoracoabdominal movements were monitored with thoracic and abdominal strain gauges. Airflow was monitored with an oronasal thermistor per the clinical protocol. Arterial oxyhemoglobin saturation was recorded with the use of a pulse oximeter. Electrocardiogram, snoring and body position were also recorded. Recordings were manually scored according to the standard criteria. ${ }^{13}$ An episode of obstructive apnea was defined as an absence of airflow for at least $10 \mathrm{~s}$, in the presence of rib cage and abdominal excursions. Hypopnea was defined as a discernible reduction in airflow lasting $10 \mathrm{~s}$ or longer and associated with at least a $4 \%$ decrease in arterial oxyhemoglobin saturation, an electroencephalographic arousal, or both. The number of episodes of apnea and hypopnea per hour was referred to as the apnea hypopnea index (AHI). The mean and minimal oxygen saturation for the entire night was estimated, along with the duration of stages 1, 2, 3, and rapid eye movement (REM) of sleep; sleep fragmentation was assessed using the arousal index (AI). The sleep parameters were scored and OSA was defined according to the criteria established by the American Academy of Sleep Medicine Manual for Scoring Sleep and Associated Events. ${ }^{13}$

Morning blood samples were collected for white blood cell count (WBC) with the percentage of peripheral neutrophils (PPN), C-reactive protein (CRP), fibrinogen, and procalcitonin, from subjects who attended the TeleHealth appointment. Assays were performed in a single run by the Salem VAMC laboratory. 
The normality of continuous variables was evaluated by the Shapiro-Wilk test. Comparison of continuous data between the OS and COPD-only groups was performed using a t-test for data with normal distribution. Peripheral neutrophils, systolic blood pressure and serum procalcitonin had a skewed distribution and the difference between groups was tested by the Wilcoxon-MannWhitney test. Since BMI has a known association with OS and chronic inflammation, stratified analysis by obesity (BMI $>30 \mathrm{~kg} / \mathrm{m}^{2}$ ) was performed to assess for potential confounding. A 2 -sided p-value $<0.05$ was considered statistically significant. All analyses were performed using STATA v. 11.0 software (Stata Corporation, College Station, USA).

\section{Results}

Thirty-eight subjects with confirmed COPD were included in the study and 17 (44\%) of these were classified as OS. Seven out of the 17 subjects with OS had an excellent CPAP compliance as defined by Medicare guidelines, i.e., the use of CPAP devices for 4 or more hours per night on $70 \%$ of nights. ${ }^{14}$ The subjects' baseline characteristics are detailed in Table 1 . The 112 subjects who were eligible for the study (CAN score $>95$ and a diagnosis of COPD) but could not be reached by phone or who declined participation in the Tele-Health appointments were not demographically different from those included in the analysis.

All OS subjects had an AHI greater than 5, except 1 patient whose AHI was 4.7. This patient was diagnosed with OSA and therefore classified as OS as well, due to the evidence of snoring with arousals during REM sleep, desaturations (at least 4\%) and symptoms suggestive of OSA, such as excessive daytime sleepiness. The AI, as well as mean and lowest oxygen saturations during the sleep study are described in Table 2. There was a significant difference $(\mathrm{p}=0.002)$ in PPN values in OS patients $(73 \pm 9.3)$ vs COPDonly patients $(60 \pm 14.9)$, regardless of whether OS subjects were compliant with the CPAP therapy $(p=0.03)$ or not $(\mathrm{p}=0.005)$. There was no difference in the PPN values between CPAP-compliant and noncompliant OS subjects $(\mathrm{p}=0.91)$. The association of PPN and OS remained significant for obese subjects $(\mathrm{p}=0.04)$ and non-obese subjects $(\mathrm{p}=0.03)$. The serum inflammatory markers, WBC, CRP, fibrinogen, and procalcitonin were similar between the OS subjects and COPD-only subjects (Table 3), regardless of their compliance $(\mathrm{p}>0.11)$ or noncompliance ( $p>0.17$ ) with the CPAP therapy. No differences in the severity of COPD, baseline comorbidities, smoking, or home oxygen therapy (HOT) use (at night, during the day or continuously) were found between OS subjects and COPD-only subjects (Table 1). Body mass index was higher in the OS group (Table 2). Body mass index, COPD severity, smoking, and HOT use (diurnal, nocturnal or continuous) were not associated with PPN (p-values $>0.2$ ).
Table 1. Subjects' baseline characteristics

\begin{tabular}{|l|c|c|c|}
\multicolumn{1}{|c|}{ Variable } & $\begin{array}{c}\text { OS group } \\
\text { (mean } \pm \text { SEM) }\end{array}$ & $\begin{array}{c}\text { COPD-only } \\
\text { group } \\
\text { (mean } \pm \text { SEM) }\end{array}$ & p-value \\
\hline Number & 17 & 21 & NS \\
$\begin{array}{l}\text { Demographics } \\
\text { age [years] } \\
\text { active smoking [\%] }\end{array}$ & $69 \pm 1.3$ & $74 \pm 1.9$ & NS \\
BMI [kg/m²] & 23 & 33 & NS \\
\hline $\begin{array}{l}\text { Hemodynamics } \\
\text { systolic blood pressure } \\
\text { [mm Hg] } \\
\text { diastolic blood pressure } \\
\text { [mm Hg] } \\
\text { heart rate [beats/min] }\end{array}$ & $32 \pm 1.9$ & $27 \pm 1.3$ & 0.02 \\
\hline CCl Score & $74 \pm 2$ & $69 \pm 3$ & NS \\
\hline Framingham Score & $6 \pm 0.31$ & $511 \mathrm{~N}$ & NS \\
\hline
\end{tabular}

OS - overlap syndrome; CCI - Charlson Comorbidity Index; COPD - chronic obstructive pulmonary disease; BMI - body mass index; SEM - standard error of the mean; NS - not significant.

Table 2. Description of the severity of COPD and OSA disease

\begin{tabular}{|c|c|c|c|}
\hline Variable & $\begin{array}{l}\text { OS group } \\
\text { (mean } \pm S D)\end{array}$ & $\begin{array}{l}\text { COPD-only } \\
\text { group } \\
\text { (mean } \pm S D)\end{array}$ & $p$-value \\
\hline \multicolumn{4}{|c|}{ COPD } \\
\hline FEV1 [L] & $1.6 \pm 0.6$ & $1.6 \pm 0.5$ & NS \\
\hline FEV1 [\%] & $48 \pm 17$ & $52 \pm 13$ & NS \\
\hline $\begin{array}{l}\text { mild [\%] } \\
\text { moderate [\%] } \\
\text { severe [\%] }\end{array}$ & $\begin{array}{l}43 \\
43 \\
12\end{array}$ & $\begin{array}{l}60 \\
30 \\
10\end{array}$ & $\begin{array}{l}\text { NS } \\
\text { NS } \\
\text { NS }\end{array}$ \\
\hline \multicolumn{4}{|c|}{ OSA } \\
\hline $\begin{array}{l}\text { AHI } \\
\text { CPAP compliant, } n \\
\text { arousal index [events/h] } \\
\text { mean oxygen saturation [\%] } \\
\text { lowest oxygen saturation [\%] }\end{array}$ & $\begin{array}{c}17 \pm 6 \\
7 \\
28.6 \pm 16 \\
81 \pm 6.3 \\
91 \pm 1.7\end{array}$ & $\begin{array}{l}\text { NA } \\
\text { NA } \\
\text { NA } \\
\text { NA } \\
\text { NA }\end{array}$ & NA \\
\hline MRC Dyspnea Score & $2.8 \pm 1.2$ & $2.9 \pm 1$ & NS \\
\hline BMI & $32 \pm 8.1$ & $27 \pm 6.4$ & NA \\
\hline \multicolumn{4}{|c|}{ HOT } \\
\hline $\begin{array}{l}\text { diurnal [\%] } \\
\text { nocturnal [\%] } \\
\text { continuous [\%] }\end{array}$ & $\begin{array}{c}35 \\
35 \\
0\end{array}$ & $\begin{array}{c}38 \\
38 \\
0\end{array}$ & $\begin{array}{l}\text { NS } \\
\text { NS } \\
\text { NS }\end{array}$ \\
\hline $\begin{array}{l}\text { Steroid use } \\
\text { inhaled, n } \\
\text { oral steroid, n }\end{array}$ & $\begin{array}{c}10 \\
2\end{array}$ & $\begin{array}{l}9 \\
0\end{array}$ & $\begin{array}{l}\text { NS } \\
\text { NS }\end{array}$ \\
\hline
\end{tabular}

COPD - chronic obstructive pulmonary disease; FEV1 (L) - forced expiratory volume in $1 \mathrm{~min}$ in liters; FEV1 (\%) - forced expiratory volume in $1 \mathrm{~min}$ as a percentage compared to controls; OSA - obstructive sleep apnea; $\mathrm{AHI}$ - apnea-hypopnea index; CPAP - positive airway pressure therapy; MRC Dyspnea Score - Medical Research Council Dyspnea Score; $\mathrm{BMI}$ - body mass index; HOT - home oxygen therapy; SD - standard deviation; NS - not significant; NA - not applicable.

\section{Discussion}

In a clinic-based sample of community-dwelling subjects with a high level of overall comorbidity, we found that PPN is higher in OS subjects compared to COPD-only subjects, regardless of their compliance with the CPAP therapy. 
Table 3. Description of the inflammatory markers

\begin{tabular}{|c|c|c|c|c|}
\hline \multirow{2}{*}{ Inflammatory marker } & \multicolumn{2}{|c|}{ OS group (mean $\pm \mathrm{SD}$ ) } & \multirow{2}{*}{ COPD-only (mean $\pm S D$ ) } & \multirow{2}{*}{$\mathrm{p}$-value } \\
\hline & CPAP compliant & CPAP noncompliant & & \\
\hline $\begin{array}{l}\text { White blood cells [cells/mm³] } \\
\text { PPN [\%] } \\
\text { C-reactive protein }[\mathrm{mg} / \mathrm{L}] \\
\text { Fibrinogen }[\mathrm{mg} / \mathrm{dL}] \\
\text { Procalcitonin }[\mathrm{mg} / \mathrm{mL}]\end{array}$ & $\begin{array}{c}8.8 \pm 4.1 \\
72 \pm 1.7^{*} \\
9.6 \pm 4.9 \\
403 \pm 58 \\
0.06 \pm 0.03\end{array}$ & $\begin{array}{c}8.7 \pm 2.3 \\
73 \pm 9.5^{* *} \\
5.7 \pm 3.8 \\
442 \pm 114 \\
0.07 \pm 0.04\end{array}$ & $\begin{array}{c}7.8 \pm 1.8 \\
60 \pm 14.9 \\
9.2 \pm 12.4 \\
397 \pm 70.6 \\
0.05 \pm 0.007\end{array}$ & $\begin{array}{l}\text { NS } \\
<0.03 \\
\text { NS } \\
\text { NS } \\
\text { NS }\end{array}$ \\
\hline
\end{tabular}

* PPN in CPAP compliant vs COPD subjects, $p=0.03$; * PPN in CPAP noncompliant vs COPD subjects, $p=0.005$ ); OS - overlap syndrome;

COPD - chronic obstructive pulmonary disease; PPN - peripheral neutrophils; CPAP - positive airway pressure therapy; SD - standard deviation.

The severity of COPD was similar between groups and, moreover, was not associated with PPN. These results suggest that the additive adverse effect of OSA is associated with increased PPN in OS and they support further research on neutrophil-related inflammation and cardiovascular disease (CVD) in these subjects.

The role of increased inflammation in sleep disordered breathing $(\mathrm{SDB})$ has been receiving increased attention recently. Systemic inflammation measured by standard inflammatory markers such as CRP and interleukin-6 (IL-6) has been studied in both COPD and OSA and supports causation between inflammation and early atherosclerosis and association between inflammation and worse outcomes. ${ }^{15-17}$ Data on the inflammatory response in OS is currently limited to only a few studies to date. Nural et al. demonstrated that the serum CRP level was higher in the OS group than the COPD-only or OSAonly groups. ${ }^{18}$ Mansour et al. showed that nasal CPAP decreased levels of TNF- $\alpha$, CRP and IL- 6 in these subjects. ${ }^{19}$ While no direct link has yet been demonstrated between inflammation and CVD in OS subjects, Marin et al. reported a higher number of cardiovascular deaths in subjects with untreated OS compared to treated OS, and a higher number of deaths than in those with COPD only. ${ }^{1}$ Although the concept of inflammation-related CVD in SDB is largely accepted,the evidence supporting it is difficult to find as the typical inflammatory markers, such as CRP and IL-6, are heavily confounded by obesity and smoking status. ${ }^{20}$ Therefore, other serum markers, such as neutrophils, are worth exploring given their lack of a strong connection with BMI (less than $10 \%$ of the variance of change). ${ }^{21}$ Moreover, the neutrophil count is inexpensive, reliable, easy to interpret, and ordered routinely in inpatient and outpatient settings. Whether neutrophils are different in obesity or not is controversial, as both increased percentages in African-American children and unchanged numbers in men have been reported. ${ }^{22,23}$ In addition, Dixon et al. showed that even when neutrophils change with BMI in adults, this change was small and accounted for less than $10 \%$ of the variance of baseline counts. ${ }^{21}$ In our study, OS subjects had a significantly higher BMI than the COPD-only subjects, but BMI was not associated with PPN.

The detailed mechanism by which neutrophils contribute to inflammation-mediated CVD is yet unknown, but possibly related to an abnormal antiapoptotic response as a response to hypoxia, with increased neutrophil survival, migration towards endothelial injury and augmentation of the atherosclerotic nidus. ${ }^{24}$ Recent evidence suggests that hypoxia is associated with delayed apoptosis of neutrophils in COPD and decreases apoptosis in OSA subjects, a process that may induce vascular damage through the previously mentioned biochemical and hematological mechanisms. ${ }^{24,25}$ Overall, the apoptosis of neutrophils represents a fundamental and intricate process of intracellular death/survival signaling pathways whose overall balance aims at containing the inflammatory response. ${ }^{26}$ In OSA and COPD subjects, antiapoptotic myeloid cell leukemia 1 (Mcl-1) is upregulated and the proapoptotic Bax or Bak molecules are downregulated. ${ }^{24,27}$ This concept becomes especially important for OS subjects who have both COPD and OSA. In addition to these quantitative changes, in both OSA and COPD, the neutrophils undergo qualitative changes being "primed" for an enhanced respiratory burst and ROS production, thus activating the inflammatory cascade and leading to the development of impaired endothelial function. ${ }^{28-30}$ These findings suggest congruently

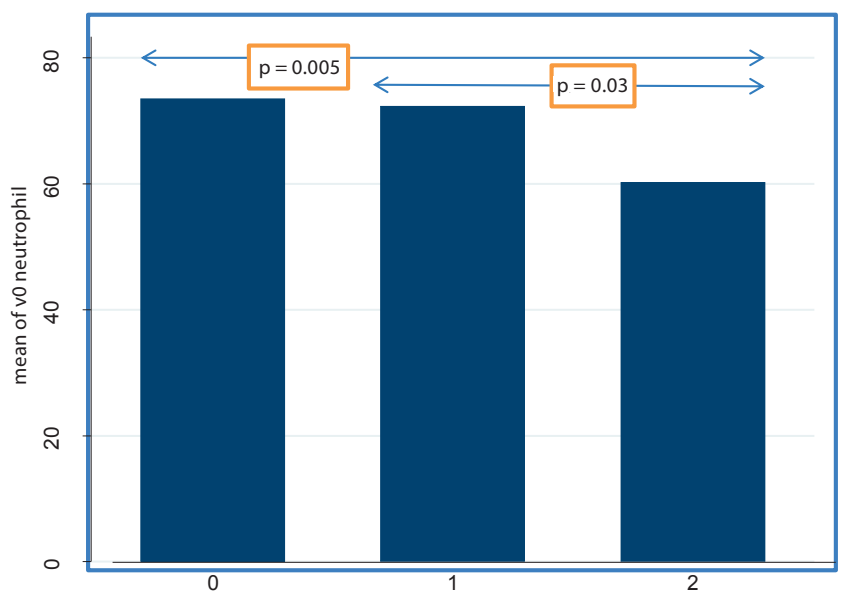

Fig. 1. Peripheral neutrophils (PPN) in subjects with OS vs COPD-only

OS - overlap syndrome; CPAP - positive airway pressure therapy; COPD - chronic obstructive pulmonary disease; group 0 - OS subjects noncompliant with CPAP; group 1 - OS subjects compliant with CPAP; group 2 - COPD-only subjects; PPN was higher in subjects with OS than COPD regardless of CPAP compliance. 
that the study of neutrophils in OS is clinically significant given their participation in the pathophysiology of CVD and the minimal confounding effects of obesity.

To the best of our knowledge, our study is the first to compare PPN in OS subjects to COPD-only subjects, thus providing important clinical results that invite larger studies on neutrophil-related inflammation in these subjects. One benefit of the study is that both groups of subjects were followed in the same outpatient clinic and had a similar degree of comorbidity (CCI and FH score), smoking and corticosteroid use (inhaled and oral). The severity of COPD was not different between the groups and was not associated with PPN.

To date, the only 2 randomized, controlled studies that explored the effect of CPAP therapy on systemic inflammation disagreed on their conclusions. ${ }^{31-33}$ Drager et al. showed that the systemic inflammation in moderate to severe OSA was not influenced by short-term CPAP therapy. ${ }^{32}$ Although our study did not show a difference in PPN between CPAP-compliant and noncompliant OS subjects, it also had the same limitations as the other previous studies, i.e., a small sample size and variation in CPAP therapy duration and compliance.

Our study has a few other limitations. Firstly, we included subjects who were on HOT for chronic hypoxia or CPAP therapy for OSA. However, treatment with oxygen would bias the results against an association with PPN between the OS and COPD-only subjects. Secondly, since we used the CAN score as a screening tool, our study included a population that had other complex chronic illnesses and, as such, our results might not be applicable to all OS subjects. However, as the CAN score refers to the likelihood of hospitalization or death of a patient within a 1-year period, our results are applicable to the large population of high-risk OS subjects. In addition, our subjects were evenly distributed between the severity levels of COPD disease, with a majority of those having mild to moderately severe OSA, thus matching a large cohort of subjects with OS. Because we limited participants to those with COPD and a high CAN score, our study resulted in a small sample size. For this reason, these findings cannot be generalized to the broader community-based sleep subjects. Comparison to CRP was limited due to the lack of a high-sensitivity CRP (hs-CRP) assay.

In conclusion, our study of a small sample of subjects with COPD and severe comorbidities suggests that those with OS have higher PPN than those with COPD alone, regardless of their compliance status with the CPAP therapy. Future research in neutrophil-related inflammation and CVD in a larger and more diverse group of OS subjects is needed. If our results are confirmed in a larger study, they could be used to motivate OS subjects to improve their lifestyles (e.g., smoking cessation, dietary modification, exercise, and weight loss) or to comply with drug therapies aimed at reducing CVD.

\section{References}

1. Marin J, Soriano J, Carrizo SJ, Boldova A, Celli BR. Outcomes in patients with COPD and OSA. Am J Respir Crit Care Med. 2010;182(3):325-331.

2. Lopez-Acevedo M, Torres-Palacios A, Elena Ocasio-Tascón M, Campos-Santiago Z, Rodríguez-Cintrón W. Overlap syndrome: An indication for sleep studies? Sleep Breath. 2009;13(4):409-413.

3. McNicholas WT. Chronic obstructive pulmonary disease and obstructive sleep apnoea: The overlap syndrome. J Thorac Dis. 2016;8(2): 236-242.

4. Madjid M, Awan I, Willerson JT, Casscells SW. Leukocyte count and coronary heart disease: Implications for risk assessment. J Am Coll Cardiol. 2004;44(10):1945-1956.

5. Harlan JM, Killen PD, Harker LA, Striker GE, Wright DG. Neutrophilmediated endothelial injury in vitro mechanisms of cell detachment. J Clin Invest. 1981;68(6):1394-1403.

6. Mehta J, Dinerman J, Mehta P, et al. Neutrophil function in ischemic heart disease. Circulation. 1989;79(3):549-556.

7. Chen J, Chen MH, Li S, et al. Usefulness of the neutrophil-to-lymphocyte ratio in predicting the severity of coronary artery disease: A Gensini score assessment. J Atheroscler Thromb. 2014;21(12):1271-1282.

8. Green SM, Vowels J, Waterman B, Rothrock SG, Kuniyoshi G. Leukocytosis: A new look at an old marker for acute myocardial infarction. Acad Emerg Med. 1996;3(11):1034-1041.

9. Office of Informatics and Analytics Veterans Health Administration. Care Assessment Need (CAN) Score and the Patient Care Assessment System (PCAS): Tools for Care Management. http://www.hsrd. research.va.gov/for_researchers/cyber_seminars/archives/713notes.pdf. Accessed June 8, 2018.

10. Hall WH, Ramachandran R, Narayan S, Jani AB, Vijayakumar S. An electronic application for rapidly calculating Charlson comorbidity score. BMC Cancer. 2004;94. doi: 10.1186/1471-2407-4-94

11. National Heart Lung and Blood Institute. Assessing Cardiovascular Risk: Systematic Evidence Review from the Risk Assessment Work Group. http://cvdrisk.nhlbi.nih.gov/calculator.asp. Accessed June 18, 2018.

12. Stenton C. The MRC breathlessness scale. Occup Med (Lond). 2008;58(3):226-227.

13. Kales A, Rechtschaffen A, eds. 13A Manual of Standardized Terminology and Techniques and Scoring System for Sleep Stages of Human Subjects. Bethesda, MD: National Institute of Neurological Diseases and Blindness, Neurological Information Network; 1968.

14. Department of Health and Human Services, Centers for Medicare and Medicaid Services. Continuous and Bi-level Positive Airway Pressure(CPAP/BPAP) Devices: Complying with Documentation and Coverage Requirements. https://www.cms.gov/Outreach-and-Education/ Medicare-Learning-Network-MLN/MLNProducts/downloads/PAP_DocCvg_FactSheet_ICN905064.pdf. Accessed June 18, 2018.

15. Nadeem R, Molnar J, Madbouly EM, et al. Serum inflammatory markers in obstructive sleep apnea: A meta-analysis. J Clin Sleep Med. 2013;9(10):1003-1012.

16. Agustí A, Edwards LD, Rennard SI, et al.; Evaluation of COPD Longitudinally to Identify Predictive Surrogate Endpoints (ECLIPSE) Investigators. Persistent systemic inflammation is associated with poor clinical outcomes in COPD: A novel phenotype. PLoS One. 2012;7(5):e37483. doi: 10.1371/journal.pone.0037483

17. Guven SF, Turkkani MH, Ciftci B, Ciftci TU, Erdogan Y. The relationship between high-sensitivity C-reactive protein levels and the severity of obstructive sleep apnea. Sleep Breath. 2012;16(1):217-221.

18. Nural S, Günay E, Halici B, Celik S, Ünlü M. Inflammatory processes and effects of continuous positive airway pressure (CPAP) in overlap syndrome. Inflammation. 2013;36(1):66-74.

19. Mansour $\mathrm{H}$, Fathy $\mathrm{A}$, Aref $\mathrm{H}$. Effect of nasal continuous positive airway pressure on inflammatory mediators inpatients with overlap syndrome. Egyptian Journal of Ear, Nose, Throat and Allied Sciences. 2011;12:99-104.

20. Quan SF, Gersh BJ. Cardiovascular consequences of sleep-disordered breathing: Past, present and future report of a workshop from the National Center on Sleep Disorders Research and the National Heart, Lung, and Blood Institute. Circulation. 2004;109(8):951-957.

21. Dixon JB, O'Brien PE. Obesity and the white blood cell count: Changes with sustained weight loss. Obes Surg. 2006;16(3):251-257.

22. Xu X, Su S, Wang X, et al. Obesity is associated with more activated neutrophils in African American male youth. Int J Obes (Lond). 2015;39(1):26-32. 
23. Ilavská $S$, Horváthová $M$, Szabová $M$, et al. Association between the human immune response and body mass index. Hum Immunol. 2012;73(5):480-485.

24. Zhang J, He J, Xia J, Chen Z, Chen X. Delayed apoptosis by neutrophils from COPD patients is associated with altered bak, bcl-xl, and mcl-1 mRNA expression. Diagn Pathol. 2012;7:65. doi: 10.1186/17461596-7-65

25. Dyugovskaya L, Polyakov A, Lavie P, Lavie L. Delayed neutrophil apoptosis in patients with sleep apnea. Am J Respir Crit Care Med. 2008;177(5):544-554.

26. Dyugovskaya L, Polyakov A, Ginsberg D, Lavie P, Lavie L. Molecular pathways of spontaneous and TNF-a-mediated neutrophil apoptosis under intermittent hypoxia. Am J Respir Cell Mol Biol. 2011;45(1):154-162.

27. Dyugovskaya L, Polyakov A, Cohen-Kaplan V, Lavie P, Lavie L. Bax/Mcl-1 balance affects neutrophil survival in intermittent hypoxia and obstructive sleep apnea: Effects of p38MAPK and ERK1/2 signalling. J Trans/ Med. 2012;10:211. doi: 10.1186/1479-5876-10-211

28. Schulz R, Mahmoudi S, Hattar K, et al. Enhanced release of superoxide from polymorphonuclear neutrophils in obstructive sleep apnea. Impact of continuous positive airway pressure therapy. Am J Respir Crit Care Med. 2000;162(2 Pt 1):566-570.
29. Stockley JA, Walton GM, Lord JM, Sapey E. Aberrant neutrophil functions in stable chronic obstructive pulmonary disease: The neutrophil as an immunotherapeutic target. Int Immunopharmacol. 2013;17(4):1211-1217.

30. Pilkauskaite G, Miliauskas S, Sakalauskas R. Reactive oxygen species production in peripheral blood neutrophils of obstructive sleep apnea patients. ScientificWorldJournal. 2013;12:421763. doi: $10.1155 / 2013 / 421763$

31. Xie X, Pan L, Ren D, et al. Effects of continuous positive airway pressure therapy on systemic inflammation in obstructive sleep apnea: A meta-analysis. Sleep Med. 2013;14(11):1139-1150.

32. Drager LF, Bortolotto LA, Figueiredo AC, et al. Effects of continuous positive airway pressure on early signs of atherosclerosis in obstructive sleep apnea. Am J Respir Crit Care Med. 2007;176(7):706-712.

33. Kohler M, Ayers L, Pepperell J, et al. Effects of continuous positive airway pressure on systemic inflammation in patients with moderate to severe obstructive sleep apnoea: A randomized controlled trial. Thorax. 2009;64(1):67-73. 